\title{
Estudio del mercado importador de productos para laboratorio en el Perú
}

\author{
Study of the import market of laboratory products in the Peru
}

DOI: $10.46932 / \mathrm{sfjdv} 3 \mathrm{n} 1-111$

Received in: Jan 30st, 2021

Accepted in: Feb 1th, 2022

\author{
Franklin Duarte Cueva \\ Master en Responsabilidad Social Empresarial and \\ Magíster en Administración de Empresas y Licenciado en Administración \\ Institution: Pontificia Universidad Católica del Perú \\ Address: Av. Universitaria 1801, San Miguel 15088, Lima, Perú \\ E-mail: fduarte@pucp.edu.pe
}

\section{RESUMEN}

La investigación analiza el mercado importador de productos para laboratorio en el Perú desde un enfoque descriptivo. Este mercado funciona desde hace más de 70 años, abasteciendo a los sectores salud, agroindustria, minero, pesquero y educación, principalmente, pero existe escasa información. El objetivo de la investigación es describir el funcionamiento del mercado importador de productos para laboratorio en Perú y conocer, de modo general, cómo fue afectado por la pandemia. Se estudia los comienzos de la industria, las principales empresas participantes y su evolución; se hace un análisis del entorno general y sectorial; se revisan las importaciones y ventas de las principales empresas, principales productos importados por país de origen y el canal de distribución. El mercado de productos para laboratorio es un mercado industrial en proceso de maduración, el cual ha sido afectado temporalmente por la pandemia.

Palabras clave: entorno general, entorno sectorial, importaciones, laboratorio, mercado industrial, pandemia.

\begin{abstract}
The research analyzes the import market for laboratory products in Peru from a descriptive approach. This market has been operating for more than 70 years, supplying the health, agribusiness, mining, fishing and education sectors, mainly, but there is little market information. The objective of the research is to describe the functioning of the import market for laboratory products in Peru and to know in a general way and how it was affected by the pandemic. The beginnings of the industry, the main participating companies and their evolution are studied; an analysis of the general and sectorial environment is made; imports and sales of the main companies, main imported products by country of origin and distribution channel are reviewed. The laboratory products market is an industrial market in maturing process, which has been temporarily affected by the pandemic.
\end{abstract}

Keywords: general environment, imports, industrial environment, industrial market, laboratory, pandemic. 


\section{INTRODUCCIÓN}

\subsection{LABORATORIO Y TIPOS}

En general, un laboratorio es un espacio físico específicamente diseñado, acondicionado y abastecido de materiales, instrumentos y personal especializado, para hacer experimentos con el fin de investigar, probar, verificar, controlar y/o proyectar resultados en diferentes campos del conocimiento. Evidentemente, todos los laboratorios tienen objetivos, políticas y procedimientos establecidos, según su propósito de investigación. Los laboratorios pueden clasificarse bajo distintos criterios. Para esta investigación, usaremos el criterio de la naturaleza de la actividad económica desarrollada por el laboratorio. Así, podemos hablar de laboratorios de control de calidad, laboratorios de análisis clínicos, laboratorios de investigación y desarrollo, laboratorios de análisis de suelos, laboratorios de análisis de aguas, laboratorios de química y laboratorios de biología. Estos laboratorios son los principales clientes de las empresas importadoras.

\subsection{ANTECEDENTES DE LA INDUSTRIA}

La falta de producción nacional debido a la escasez de tecnología, recursos financieros y estrechez del mercado interno, favoreció que inmigrantes extranjeros emprendieran las primeras aventuras de creación de empresas importadoras de productos para laboratorio, las cuales se abastecían de Europa y los Estados Unidos. La primera empresa que se constituyó en el mercado fue H.W. Kessel S.A., en 1950, la cual fue fundada por un residente de origen alemán de nombre Helmut Wolfgang Kessel S.A. Posteriormente, en 1957, fue constituida la firma AHSECO S.A. (American Hospital Scientific Equipment del Perú). En 1960, un inmigrante de origen belga llamado Alexander Kossodo creó la empresa A.H. Kossodo S.A. En 1962, el inmigrante alemán Heinz Abendroth fundó la empresa CIMATEC S.A. (Compañía Importadora de Materiales Técnicos para Laboratorio). En 1983, fue fundada la firma A. Jaime Rojas Representaciones Generales S.A., de origen netamente nacional. Estas empresas han concentrado la mayor participación de mercado durante décadas. Por otra parte, se promulgaron normas legales destinadas a garantizar la libertad de comercio exterior e interior como condición fundamental para el desarrollo del país. Mediante el Decreto Legislativo 668 (1991), cuyo artículo 2 establecía "El Estado garantiza a los agentes económicos el libre acceso a la adquisición, transformación y comercialización de bienes, tanto finales como insumos y materias primas, y prestación de servicios”, se promovió un mercado más abierto al exterior. La siguiente tabla registra le experiencia de las empresas en el mercado. 
Tabla 1: Principales firmas importadoras de productos para laboratorio en el mercado peruano, según fecha de fundación

\begin{tabular}{|c|l|c|c|c|}
\hline Ranking & \multicolumn{1}{|c|}{ Razón social } & $\begin{array}{c}\text { Fecha de } \\
\text { fundación }\end{array}$ & $\begin{array}{c}\text { Tiempo en } \\
\text { el mercado }\end{array}$ & $\begin{array}{c}\text { Empleados a } \\
\text { enero 2021 }\end{array}$ \\
\hline $1^{\mathbf{o}}$ & H.W. Kessel S.A. & 07.12 .1950 & 71 años & 60 \\
\hline $2^{\mathbf{o}}$ & AHSECO Perú S.A. & 23.10 .1957 & 64 años & 51 \\
\hline $3^{\mathbf{o}}$ & Kossodo S.A.C. & 12.05 .1960 & 61 años & 101 \\
\hline $4^{\mathbf{o}}$ & CIMATEC S.A. & 01.06 .1962 & 59 años & 44 \\
\hline $5^{\mathbf{o}}$ & A. Jaime Rojas Reps. Generales S.A. $\left(^{*}\right)$ & 01.04 .1983 & 38 años & 121 \\
\hline
\end{tabular}

(*) A. Jaime Rojas Representaciones Generales S.A.

Fuente: elaborada con información de la Oficina Nacional de Registros Públicos y SUNAT.

Las importaciones destinadas a los clientes del mercado interno de productos para laboratorio corresponden al régimen aduanero de importación para el consumo, el cual según el Decreto Legislativo 1053 denominado Ley General de Aduanas (2008), es definido como el régimen aduanero que permite ingresar mercancías al territorio aduanero para su consumo, luego del pago o garantía según corresponda, de los derechos arancelarios y demás impuestos aplicables, así como el pago de los recargos y multas que hubieren, y del cumplimiento de las formalidades y otras obligaciones aduaneras. Esto implica que las mercancías permanecerán de forma definitiva en el territorio aduanero.

El mercado importador de productos para laboratorio es un mercado industrial porque las empresas importadoras abastecen de productos a otras empresas que los utilizan en la producción de mercancías, prestación de servicios o los revenden. El mercado industrial también es conocido con el nombre de mercado empresarial, en este sentido, Kotler (2012) explica que el mercado empresarial está conformado por todas las organizaciones que adquieren bienes y servicios para utilizarlos en la producción de otros productos o servicios que se venden, alquilan o suministran a otras entidades.

Valencia, J. (comunicación personal, 10 marzo 2016), refiere que este mercado comenzó por la visión de emprendedores de ascendencia extranjera, quienes usaron sus relaciones comerciales en sus países de origen. Los ayudó, ser nativos de idiomas extranjeros, la disciplina para hacer negocios y la confianza que construyeron en el mercado interno. Por otro lado, la tendencia creciente del PBI expresada en un ascendente consumo interno e incremento de las exportaciones, impulsó la creación de otras empresas importadoras que, a través del tiempo, se han diversificado y expandido sus negocios en el mercado nacional. (Navas, comunicación personal, 25 mayo 2016) opina que el mercado de productos para laboratorio es cada vez más competitivo y demanda un alto nivel de profesionalismo así como elevada calidad de servicio, para proporcionar una experiencia distintiva al cliente.

\subsection{TAMAÑO EMPRESARIAL DE LAS PRINCIPALES EMPRESAS SEGÚN LAS NORMAS}

\section{LEGALES}

En los diferentes países, los gobiernos buscan promover el crecimiento de las empresas y promulgan normas para determinar el tamaño de las empresas con el fin de otorgar ciertos beneficios, por 
otro lado, tratan de ajustar sus políticas tributarias y mejorar la recaudación. En este contexto, las principales empresas del mercado importador de productos para laboratorio han evolucionado positivamente como resultado del crecimiento del producto bruto interno, el aumento de su competitividad, el aprendizaje logrado a través de la curva de la experiencia y las estrategias implementadas.

Tabla 2: Principales firmas del mercado importador de productos para laboratorio en el Perú según domicilio, volumen de ventas y tamaño empresarial

\begin{tabular}{|l|c|c|c|}
\hline Razón Social & $\begin{array}{c}\text { Domicilio fiscal por } \\
\text { distrito }\end{array}$ & $\begin{array}{c}\text { Volumen de } \\
\text { ventas anual }(*)\end{array}$ & $\begin{array}{c}\text { Ley 30056 } \\
\text { (Año 2020) }\end{array}$ \\
\hline H.W. Kessel S.A.C. & Miraflores & $27,481,567$ & Gran empresa \\
\hline AHSECO Perú S.A. & Lince & $22,000,000$ & Gran empresa \\
\hline Kossodo S.A.C. & Cercado de Lima & $26,371,399$ & Gran empresa \\
\hline CIMATEC S.A. & Cercado de Lima & $16,499,990$ & Gran empresa \\
\hline A. Jaime Rojas Reps. Generales S.A. & Barranco & $51,143,000$ & Gran empresa \\
\hline
\end{tabular}

(*) Ventas en moneda nacional para el año 2020.

Fuente: elaborada con datos de Normas Legales del Diario El Peruano, DIME y Top Online.

La ley 30056, estableció que se considera como medianas empresas a aquellas compañías cuyo volumen de ventas anual se encuentre en el rango de 1700 a 2300 UIT (Unidad Impositiva Tributaria), las empresas que facturan por encima de ese rango, son catalogadas como grandes empresas. Para el año 2020, el valor de la UIT, según el Decreto Supremo No 380-2019-EF, ascendió a S/. 4,300 (\$1228.6); entonces 1700 UIT es igual a S/.7’310,000 (\$2’088,571.4) y 2300 UIT es igual a S/.9’890,000 ((\$2’825,714.3). Con esta información, se concluye que las cinco principales empresas importadoras de productos para laboratorio en el Perú, se clasifican como grandes empresas.

\section{MARCO TEÓRICO}

Esta parte está dedicada a explorar el entorno general y sectorial que enfrentan las firmas importadoras de productos para laboratorio, según autores referentes, con el objetivo de comprender el funcionamiento del mercado.

\subsection{ANÁLISIS DEL ENTORNO GENERAL}

La relevancia de los factores del entorno general puede ser evaluada usando el análisis PESTEL, un acrónimo para los seis principales componentes del macro ambiente: factores políticos, factores económicos, factores sociales, factores tecnológicos, factores ambientales y factores legales (Thompson et al, 2015). El análisis PESTEL es una herramienta de planeamiento estratégico que permite evaluar el impacto del comportamiento de las variables del entorno general, en el desempeño de un negocio en marcha o de un nuevo negocio. Así, tenemos: 


\section{a) Variables políticas}

-Sistema de gobierno: el Perú es un país que mantiene el sistema democrático con elecciones generales para la presidencia y el congreso por períodos de cinco años. Se respetaron estos procesos desde 1980, sin embargo, a partir del año 2017, el mecanismo de la vacancia ha sido utilizado arbitrariamente por el congreso para tratar de cortar los períodos presidenciales de los tres últimos presidentes de la república. Esta situación, es una amenaza para las empresas participantes en la industria pues genera incertidumbre.

-Estabilidad política: En las últimas elecciones generales, resultó electo el profesor Pedro Castillo candidato del partido político Perú Libre por una escasa cantidad de votos. Este hecho ha causado polaridad en la ciudadanía y una buena parte de los congresistas electos en las mismas elecciones. La situación ha generado que haya cuatro gabinetes de ministros en solo seis meses de gobierno, algo inaudito en la historia política peruana. El enfrentamiento entre el congreso y el ejecutivo produce gran inestabilidad política. Esto representa una amenaza para las empresas en general.

\section{b) Variables económicas}

Las principales variables influyentes, cuyos datos se muestran en el anexo 2, son:

\section{-Producto Bruto Interno}

Se analizó la variación porcentual anual para comprender la dinámica del crecimiento de la economía durante el período 2001-2020, de acuerdo con los datos del Banco Central de Reserva del Perú. Durante dicho período se observó una tendencia creciente hasta el año 2019. En 2020, el surgimiento de la pandemia contrajo la economía mundial. En los períodos precedentes 2001-2019 hubo un crecimiento sostenido del PBI, lo cual influyó en los mayores volúmenes importados y comercializados en la industria. La situación actual, por la aparición de nuevas variantes del coronavirus y la contracción de la demanda de productos para laboratorio, implica una amenaza para las empresas de la industria.

\section{-Tipo de cambio}

La fluctuación del tipo de cambio ha reflejado variaciones en la cotización del dólar, que es la moneda comúnmente usada por los importadores de la industria. En el período 2001-2020, de acuerdo con los datos del Banco Central de Reserva del Perú, la tendencia era hacia una relativa estabilidad del tipo de cambio, sin embargo, debido a la recesión económica mundial y la fuerte inestabilidad política, el tipo de cambio se ha devaluado significativamente, generando un encarecimiento del costo de compra de los productos importados, lo cual afecta los márgenes de utilidad de los importadores que facturan en moneda nacional. En general, esta situación es una amenaza para las empresas. 


\section{-Inflación}

Durante el período 2001-2020, la tasa de inflación anual, según datos del Banco Central de Reserva del Perú registró tasas menores al 4\% anual, lo cual no afectó el poder de compra de las empresas clientes y tampoco propició la descapitalización de las empresas de la industria. Sin embargo, en 2021, la tasa de inflación creció significativamente hasta 6.4\%, impulsada por la devaluación y las expectativas de los agentes económicos. Este hecho puede constituir una amenaza para las empresas.

\section{-Tasa de interés}

Durante el período 2001-2020, la evolución de la tasa de interés activa en moneda nacional, muestra una marcada tendencia de reducción consecutiva, lo cual genera una disminución de los gastos financieros para las empresas que trabajan con deuda bancaria, como es el caso de las principales empresa importadoras de productos para laboratorio. Esta situación representa una oportunidad para dichas empresas porque pueden mejorar su rentabilidad y optimizar las estrategias de fijación de precios.

\section{c) Variables sociales:}

-Delincuencia: los índices de delincuencia respecto a extorsiones, hurto, asalto y robo, y secuestro han crecido hasta niveles nunca antes vistos, lo cual obliga a realizar mayores gastos a las empresas para protegerse de los tentáculos de las mafias y bandas que operan en diferentes sectores de la capital así como en provincias. La delincuencia también genera un daño psicológico a los empresarios, por lo cual se la considera una amenaza.

-Protestas sociales: las movilizaciones en el campo, en contra de la industria minera principalmente, influyen negativamente en los procesos de compra y suministro de los productos para laboratorio. Estos hechos constituyen una amenaza latente para las empresas de la industria porque son eventos impredecibles e incontrolables.

-Defensa del consumidor: el Instituto de Defensa de la Competencia y de la Propiedad Intelectual (INDECOPI) es el organismo estatal encargado de vigilar el cumplimiento de las ofertas de las empresas. Asimismo, la Ley 29571, denominada Código de Protección y Defensa del consumidor que creó el libro de reclamaciones y establece normas que protegen a los compradores. Esta normativa representa una oportunidad de mejora por la retroalimentación que pueden brindar los clientes de la industria.

\section{d) Variables tecnológicas:}

-Tecnologías de la información y la comunicación: se reflejan en el uso de software de negocios, utilización de Internet y los sistemas de comunicación. Los acelerados cambios tecnológicos impulsan la 
aparición de nuevo software, redes sociales, nuevos equipos de comunicaciones. La oportunidad se da para las compañías que pueden adquirir las nuevas tecnologías.

\section{e) Variables ecológicas:}

-Composición de los materiales: los proveedores extranjeros de productos para laboratorio invierten cada vez más recursos en investigación y desarrollo para utilizar insumos amigables con el medio ambiente y tienen como objetivo ayudar a reducir la huella de carbono que producen y disminuir la polución ambiental. Para ello, buscan implementar estrategias para ser productores o comercializadores socialmente responsables. Esta situación genera una oportunidad para las empresas importadoras.

-Uso de fuentes de energía: se refiere al uso de energías renovables en los procesos productivos para minimizar los niveles de emisión de dióxido de carbono. Marienfeld, G. (comunicación personal, 22 de julio 2015) declara que recogen las aguas pluviales por un recipiente colector y las limpian por ultrafiltración para reutilizarlas mediante un sistema de circulación en la producción, también, utilizan energía geotérmica limpia. Así, crean condiciones de trabajo saludables para los empleados mediante el uso de energía ecológica. La tendencia global es generar menores emisiones de gases de efecto invernadero. Esto significa una oportunidad para las firmas importadoras.

\section{f) Variables legales}

\section{-Normas aduaneras:}

Las normas marco son la Ley General de Aduanas y el Reglamento de la Ley General de Aduanas. Por otro lado, el Ministerio de Economía y Finanzas ha venido reduciendo gradualmente los aranceles aplicables a la importación de bienes para el consumo y por ende a los productos para laboratorio; por ejemplo, en 2001 la tasa arancelaria que gravaban los artículos para laboratorio era del 12\%. Según SUNAT (2020) las tasas arancelarias ad valorem que se aplican al ingreso de las mercancías extranjeras son $11 \%, 6 \%, 4 \%$ y $0 \%$. Debe precisarse que, actualmente todos los productos para laboratorio de origen importado, están afectos a tasas del 6\%. Esto genera una oportunidad para las firmas importadoras por la reducción de los costos para nacionalizar mercancías.

\section{-Normas tributarias:}

De acuerdo con Trading Economics (2022) el impuesto a la renta para las empresas en el Perú, se ha mantenido con una tasa promedio del 29,5\% anual durante el período 2017-2021. A pesar que el congreso aprobó la Ley 30296 que establecía una gradual reducción anual a partir del año 2015. En este contexto, esta variable se considera de efecto neutral 
-Leyes laborales: el Decreto Legislativo 728, Ley de productividad y competitividad laboral, es la ley marco de las normas laborales en el Perú, cuyo objetivo central era fomentar las competencias de los trabajadores para mejorar su desempeño laboral. Ha habido modificatorias, sin embargo, respecto a las contrataciones, el empleador puede administrar diversos escenarios según los tipos de contrato que establece la norma. Esta situación de flexibilidad constituye una oportunidad para los empleadores.

-Normas sanitarias: debido al surgimiento de la pandemia, el gobierno estableció normas que obligaron a un confinamiento de las personas, mediante el Decreto Supremo 044-2020-PCM. Ello condujo al cierre temporal de los negocios, lo cual implica una amenaza.

\subsection{ANÁLISIS DEL ENTORNO SECTORIAL}

Para analizar el entorno sectorial podemos usar el modelo de las cinco fuerzas. En este contexto, Magretta (2012) dice "Es el marco seminal de Porter para evaluar la competencia en cualquier industria mediante el análisis de la estructura de dicha industria". Por otro lado, respecto al modelo, Hitt et al. (2011) sostiene que el análisis de las cinco fuerzas dentro de una industria dada, permite a una empresa determinar el atractivo de una industria en términos del potencial para obtener retornos promedio o superiores al promedio. Las fuerzas establecidas por Porter (1982) son:

\section{a) Competencia potencial}

De acuerdo con Hill (2011) "los competidores potenciales son empresas que no compiten hoy en una industria, pero que tienen la capacidad de hacerlo si así lo deciden”. Según Valencia, J. (comunicación personal, 10 de marzo 2016), las tres principales barreras de entrada a la industria importadora de productos para laboratorio son las necesidades de capital, la experiencia en el negocio y la lealtad a la marca. Para comenzar un pequeño negocio en esta industria, el capital de trabajo no debe ser inferior a USD50.000. Asimismo, es esencial conocer todas las líneas de productos y sus niveles de rotación, finalmente, en esta industria hay marcas posicionadas que gozan de la preferencia de los usuarios o profesionales de los laboratorios, sin embargo, su adquisición puede estar está sujeta a temas presupuestarios. En esta industria, la competencia potencial está dada básicamente por la posibilidad que las compañías exportadoras extranjeras abran filiales en el mercado local. Esta situación puede representar una amenaza.

\section{b) Competencia actual}

David (2013) señala que la rivalidad entre empresas competidoras suele ser la más poderosa de las cinco fuerzas competitivas. Las estrategias de una empresa únicamente pueden tener éxito en la medida 
en que le proporcionen una ventaja competitiva sobre las estrategias de las empresas rivales. Esto puede verse en los niveles de venta anual de las cinco primeras firmas importadoras, los cuales son independientes del tiempo de permanencia en el mercado, tal como lo muestra la tabla siguiente. Según Valencia, J. (comunicación personal, 10 de marzo 2016), las principales cinco empresas tienen una participación de mercado aproximada del $60 \%$ de las ventas de la industria, pero el mercado tiende a atomizarse en el mediano plazo.

Tabla 3: Ventas anuales de las principales firmas del mercado de productos para laboratorio, 2001-2020 (En miles de US\$*)

\begin{tabular}{|c|c|c|c|c|c|}
\hline ÃNOS & $\begin{array}{c}\text { AHSECO Perú } \\
\text { S.A. }\end{array}$ & $\begin{array}{c}\text { CIMATEC } \\
\text { S.A. }\end{array}$ & $\begin{array}{c}\text { H.W. Kessel } \\
\text { S.A. }\end{array}$ & $\begin{array}{c}\text { Kossodo } \\
\text { S.A.C. }\end{array}$ & $\begin{array}{c}\text { A. Jaime Rojas } \\
\text { Reps. G. S.A.(/) }\end{array}$ \\
\hline 2001 & $1,537.61$ & $1,262.36$ & $1,025.64$ & $1,548.32$ & 968.66 \\
\hline 2002 & $2,246.59$ & $1,354.28$ & $1,366.69$ & $2,440.14$ & $1,491.48$ \\
\hline 2003 & $3,001.86$ & $1,416.42$ & $1,414.84$ & $2,099.72$ & $1,448.61$ \\
\hline 2004 & $3,501.63$ & $1,535.84$ & $1,468.70$ & $2,449.24$ & $1,453.82$ \\
\hline 2005 & $3,636.36$ & $1,718.76$ & $2,558.34$ & $3,068.88$ & $2,604.80$ \\
\hline 2006 & $3,700.34$ & $2,202.50$ & $2,084.36$ & $4,587.16$ & $2,880.09$ \\
\hline 2007 & $6,297.41$ & $2,399.78$ & $2,626.99$ & $4,565.43$ & $4,284.61$ \\
\hline 2008 & $7,552.95$ & $2,819.95$ & $2,944.75$ & $6,065.71$ & $5,202.55$ \\
\hline 2009 & $16,313.25$ & $2,649.79$ & $3,473.75$ & $6,230.45$ & $7,224.91$ \\
\hline 2010 & $9,739.93$ & $2,959.25$ & $3,604.95$ & $5,687.99$ & $8,616.61$ \\
\hline 2011 & $8,615.87$ & $3,095.69$ & $5,212.57$ & $5,986.83$ & $8,211.08$ \\
\hline 2012 & $8,096.97$ & $3,893.50$ & $8,193.94$ & $9,345.08$ & $10,278.79$ \\
\hline 2013 & $10,892.96$ & $3,242.22$ & $8,280.37$ & $8,088.52$ & $13,440.00$ \\
\hline 2014 & $10,551.06$ & $3,330.28$ & $6,522.54$ & $9,859.51$ & $11,519.72$ \\
\hline 2015 & $8,052.78$ & $3,952.66$ & $6,650.69$ & $10,868.56$ & $14,190.47$ \\
\hline 2016 & $7,110.11$ & $3,749.27$ & $6,905.47$ & $9,286.36$ & $13,345.27$ \\
\hline 2017 & $5,132.23$ & $3,882.30$ & $5,557.13$ & $8,714.05$ & $13,443.96$ \\
\hline 2018 & $6,350.53$ & $4,502.59$ & $5,439.05$ & $7,783.25$ & $16,547.60$ \\
\hline 2019 & $6,586.83$ & $5,089.82$ & $8,228.01$ & $7,895.63$ & $15,312.28$ \\
\hline 2020 & $8,266.86$ & $4,294.70$ & $4,850.57$ & $6,264.00$ & $17,215.14$ \\
\hline
\end{tabular}

(*) Cifras aproximadas, convertidas a dólares según el tipo de cambio promedio anual.

(/) A. Jaime Rojas Representaciones Generales S.A.

Fuente: elaborada con información de la base de datos DIME y Top Online.

En términos nominales consolidados, en el período 2001-2020, las tasas de crecimiento de las empresas fueron AHSECO (437.6\%), CIMATEC (240.2\%), Kessel (372.9\%), Kossodo (304.6\%) y Jaime Rojas (1677.2\%). El mayor crecimiento de la empresa Jaime Rojas Representaciones Generales se basa en una amplia gama de productos ofertados, la cual incluye materiales y equipamiento médico además de productos para laboratorio. Por otro lado, dicha firma se financia emitiendo bonos a través del mercado alternativo de valores (MAV) desde el año 2014, lo cual le permite acceder a financiamiento a tasas de interés competitivas. También, abrió la filial extranjera Ultraschall de Colombia SAS en 2010.

Finalmente, sobre las barreras de salida de la industria Guimbert (2010) explica "las barreras de salida de un sector son diversas. Primero los activos especializados, los que tienen poco valor de liquidación o costos elevados de transferencias o conversión”. Para la industria analizada, la principal barrera de salida son los activos especializados. En el contexto actual, la intensidad de la competencia está 
creciendo en el mercado y las barreras de salida son elevadas, esta situación representa una amenaza para las empresas.

\section{c) Poder de negociación de los clientes}

En esta industria, el poder de negociación de los clientes está determinado por los montos y frecuencia de compra de los clientes. En este sentido, las grandes empresas, que generalmente son las que más compran, tienen un alto poder de negociación y están en posición de exigir especificaciones de calidad, precios competitivos, facilidades de pago, tiempos de entrega, cantidades disponibles de productos, entre otros. Los clientes de la industria pertenecen principalmente a los sectores salud, agroindustria, minería, educación, entre otros. Esta circunstancia es una amenaza para los ofertantes de la industria.

\section{d) Poder de negociación de los proveedores}

Los principales proveedores poseen alto poder de negociación respecto a los importadores porque pueden tomar decisiones para otorgar representaciones exclusivas, distribuciones autorizadas y pueden decidir acciones de libre comercialización, según los mercados de destino. El tiempo de permanencia en el mercado les ha dado reputación y conocimiento privilegiado de la industria. Inclusive, podrían decidir integrarse verticalmente hacia adelante. En este contexto, fijan como política para sus exportaciones, montos mínimos de compra. Esta situación genera una amenaza para la industria.

Tabla 4: Principales proveedores de productos para laboratorio, a nivel global por líneas de productos, país de origen y fundación

\begin{tabular}{|l|l|l|l|l|c|}
\hline \multicolumn{1}{|c|}{ Razón social } & Sede central & \multicolumn{1}{c|}{$\begin{array}{c}\text { Línea central de } \\
\text { productos }\end{array}$} & \multicolumn{1}{|c|}{ Condición } & \multicolumn{1}{c|}{$\begin{array}{c}\text { Pá́s } \\
\text { fundación }\end{array}$} \\
\hline A. Hettich GmbH & Tutlingen & Centrífugas & Fabricante & Alemania & 1904 \\
\hline Atago Co. Ltd. & Tokio & Refractómetros & Fabricante & Japón & 1940 \\
\hline Boeco+Co GmbH & Hamburgo & Material y equipos & Intermediario & Alemania & 1929 \\
\hline Carl Zeiss A.G. & Oberkochen & Microscopios & Fabricante & Alemania & 1846 \\
\hline Corning Inc. & New York & Material de vidrio & Fabricante & USA & 1851 \\
\hline $\begin{array}{l}\text { Duran Group } \\
\text { GmbH }\end{array}$ & Wertheim & Material de vidrio & Fabricante & Alemania & 1938 \\
\hline Eppendorf AG & Hamburgo & Micropipetas & Fabricante & Alemania & 1945 \\
\hline Hanna Instrum. Inc. & R. Island & Peachímetros & Fabricante & USA & 1978 \\
\hline Kartell SpA & New York & Material de plástico & Fabricante & USA & 1949 \\
\hline Kavalier Glass AS & Sázava & Material de vidrio & Fabricante & República & 1837 \\
\hline Kimble Chase LLC & New Jersey & Material de vidrio & Fabricante & USA & 1911 \\
\hline Kyowa Optical Ltd. & Tokio & Microscopios & Fabricante & Japón & 1940 \\
\hline Memmert GmbH & Schwabach & Incubadoras & Fabricante & Alemania & 1933 \\
\hline Metrohm & Herisau & Potenciómetros & Fabricante & Suiza & 1943 \\
\hline Merck KGaA & Darmstadt & Reactivos & Fabricante & Alemania & 1668 \\
\hline $\begin{array}{l}\text { Paul Marienfeld } \\
\text { GmbH }\end{array}$ & Königshofen & Material de vidrio & Fabricante & Alemania & 1922 \\
\hline
\end{tabular}




\begin{tabular}{|l|l|l|l|l|c|}
\hline Sartorius AG & Göttingen & Balanzas & Fabricante & Alemania & 1870 \\
\hline $\begin{array}{l}\text { Sigma Aldrich } \\
\text { Corp. }\end{array}$ & Misuri & Reactivos & Fabricante & USA & 1975 \\
\hline $\begin{array}{l}\text { Thermo Fisher } \\
\text { Scientific Inc.* }\end{array}$ & Massachusetts & Espectrofótometros & Fabricante & USA & 2006 \\
\hline Thomas Sci. Inc. & New Jersey & Micropipetas & Fabricante & USA & 1900 \\
\hline Vaccubrand GmbH & Wertheim & Bombas de vacío & Fabricante & Alemania & 1985 \\
\hline $\begin{array}{l}\text { Ningbo Gamry } \\
\text { Optical Instr.** }\end{array}$ & Shanghai & Microscopios & Fabicante & China & 1997 \\
\hline
\end{tabular}

* Surgió de la fusión entre Thermo Electron y Fisher Scientific en 2006.

** El nombre de la empresa es Ningbo Gamry Optical Instrument Co., Ltd.

Fuente: elaborada con datos de entrevistas y sitios web de las empresas.

De la tabla, puede resaltarse la amplia experiencia que tienen los proveedores internacionales y su capacidad para adaptarse a las condiciones cambiantes de la industria internacional. Los datos nos indican que 16 proveedores, los cuales representan el 72.7\% del total de la muestra de empresas, tienen más de 70 años de operación en el mercado. En el caso de los proveedores chinos, son empresas que, generalmente, tienen menor tiempo de permanencia en el mercado y están aprendiendo progresivamente a competir.

\section{e) Productos sustitutos}

Hill (2005) explica que son productos o servicios de diferentes negocios o industrias que pueden satisfacer necesidades similares de los clientes. La sustitución a la que están expuestos los productos para laboratorio es prácticamente inexistente. Cada producto de laboratorio desempeña una función y uso único, y no puede ser reemplazado por otro producto de otra industria. Esto es una oportunidad para las empresas participantes.

\section{METODOLOGÍA}

La investigación es de tipo descriptiva. Hernández et al (2010), señala que los estudios descriptivos pretenden especificar las propiedades, las características y los perfiles de personas, grupos, comunidades, procesos, objetos o cualquier otro fenómeno que se someta a un análisis, buscando información de manera independiente. Según Salkind (1998), la investigación descriptiva es aquella en la cual se reseñan las características o rasgos de la situación o el fenómeno que es objeto de estudio. En la presente investigación se utiliza información cuantitativa obtenida de fuentes secundarias como son los datos estadísticos provenientes de bases oficiales e internacionales como son la Superintendencia Nacional de Aduanas y Administración Tributaria de Perú y el Centro de Comercio Internacional de Suiza. En este sentido, se analiza la información con la metodología del análisis de contenido que para Krippendorff (1990) es una técnica de investigación orientada a proponer, partiendo de ciertos datos, inferencias reproducibles y válidas que puedan aplicarse a su contexto. Por otro lado, También se utiliza información cualitativa 
obtenida de fuentes primarias, mediante el uso de entrevistas en profundidad, las cuales se aplicaron de manera individual a tres personas: un exportador alemán, una ejecutiva de una firma importadora nacional y el gerente de una empresa distribuidora local de provincia. Las proposiciones de la entrevista fueron diseñadas y validadas con la metodología del alfa de Cronbach, para lo cual se contó con el aporte de tres consultores independientes, conocedores del mercado de productos para laboratorio. Se obtuvo como coeficiente de Cronbach el valor de 0.80, el cual refleja una alta fiabilidad del instrumento. Los entrevistados se seleccionaron sobre la base de criterios considerados relevantes por el investigador (ver anexo 1).

\subsection{ANÁLISIS DE LOS VALORES IMPORTADOS POR EMPRESA SEGÚN EL INCOTERM CIF}

Para la Cámara de Comercio Internacional de Paris (2019) que regula los Incoterms 2020, bajo el Incoterm el CIF, el vendedor entrega la mercancía al comprador a bordo del buque o procura la mercancía así ya entregada. El riesgo de pérdida o daño de la mercancía se transmite cuando la mercancía está a bordo del buque. El vendedor debe contratar y pagar el flete y seguro de transporte para llevar la mercancía hasta el puerto de destino designado. A continuación se presenta los valores importados por las principales empresas de la industria.

Tabla 6: Valores importados por las principales firmas del mercado (En miles de US\$, en términos CIF)

\begin{tabular}{|c|c|c|c|c|c|}
\hline A ÑNOS & $\begin{array}{c}\text { AHSECO Perú } \\
\text { S.A. }\end{array}$ & CIMATEC S.A. & $\begin{array}{c}\text { H.W. Kessel } \\
\text { S.A.C. }\end{array}$ & $\begin{array}{c}\text { Kossodo } \\
\text { S.A.C. }\end{array}$ & $\begin{array}{c}\text { A. Jaime Rojas } \\
\text { Reps. G. S.A.* }\end{array}$ \\
\hline 2001 & 596.40 & 608.27 & 288,25 & 699.30 & 328.00 \\
\hline 2002 & $1,312.19$ & 847.23 & 559.49 & 935.55 & 857.53 \\
\hline 2003 & $1,316.03$ & $1,028.61$ & 413.12 & 890.20 & 780.30 \\
\hline 2004 & $3,287.72$ & $1,715.38$ & $1,973.10$ & $2,148.02$ & $2,428.52$ \\
\hline 2005 & $3,790.60$ & $2,450.00$ & $2,993.55$ & $3,470.88$ & $2,753.44$ \\
\hline 2006 & $2,235.84$ & $1,221.02$ & 963.34 & $2,188.03$ & $1,331.71$ \\
\hline 2007 & $4,792.15$ & $1,703.19$ & $1,109.74$ & $2,803.90$ & $3,245.64$ \\
\hline 2008 & $4,004.81$ & $1,769.64$ & $1,471.39$ & $3,055.80$ & $3,250.60$ \\
\hline 2009 & $7,360.27$ & $1,290.47$ & $1,629.45$ & $2,885.54$ & $3,742.56$ \\
\hline 2010 & $4,082.13$ & $1,529.37$ & $1,726.47$ & $3,237.94$ & $4,330.45$ \\
\hline 2011 & $6,102.66$ & $2,097.36$ & $2,672.98$ & $3,587.09$ & $5,915.67$ \\
\hline 2012 & $4,143.42$ & $2,260.99$ & $3,287.85$ & $3,729.48$ & $4,580.47$ \\
\hline 2013 & $7,613.84$ & $2,135.75$ & $4,495.41$ & $3,906.95$ & $10,708.67$ \\
\hline 2014 & $6,894.20$ & $1,806.19$ & $4,167.35$ & $5,144.22$ & $7,754.40$ \\
\hline 2015 & $2,407.44$ & $1,531.27$ & $1,611.93$ & $3,004.65$ & $3,571.04$ \\
\hline 2016 & $4,045.21$ & $1,831.81$ & $3,668.23$ & $3,394.55$ & $6,868.43$ \\
\hline 2017 & $3,606.39$ & $2,210.30$ & $3,376.67$ & $3,646.04$ & $9,712.84$ \\
\hline 2018 & $4,587.16$ & $1,832.31$ & $2,743.92$ & $4,285.49$ & $7,407.68$ \\
\hline 2019 & $4,313.82$ & $2,541.15$ & $3,758.71$ & $4,184.14$ & $5,198.25$ \\
\hline 2020 & $4,499.08$ & $2,047.78$ & $2,708.12$ & $3,081.75$ & $3,339.17$ \\
\hline
\end{tabular}

*A. Jaime Rojas Representaciones Generales S.A. vende además productos médicos.

Fuente: elaborada con datos de http://www.sunat.gob.pe 
Durante el período 2001 al 2019, se observa una tendencia relativamente fluctuante de los valores importados, en el año 2020 las importaciones sufrieron una contracción, motivada por los agentes económicos quienes adoptaron una posición más conservadora dadas las implicancias de la pandemia, la incertidumbre y la recesión económica global. Por otro lado, la desviación estándar de los valores importados en miles de dólares americanos por cada empresa, en el período 2001-2020, es como sigue 1,930.6 para AHSECO Perú; 522.1 para CIMATEC; 1,283.6 para H.W. Kessel; 1,161.9 para Kossodo y 2,894.1 para A. Jaime Rojas Representaciones Generales, la cual presenta la mayor dispersión junto con AHSECO Perú. Esta situación se refleja en los volúmenes de venta logrados.

\subsection{ANÁLISIS DE PRODUCTOS IMPORTADOS POR PARTIDA ARANCELARIA Y PAÍS}

\section{PROVEEDOR}

Antes de analizar los principales productos importados se procedió a estructurar la mezcla de productos para la industria, la cual a nivel de unidad empresarial es definida por Stanton (2007) como el conjunto de todos los productos ofrecidos a la venta por una empresa. La estructura de una mezcla de productos tiene amplitud y profundidad. Su amplitud se mide por el número de líneas de productos que comprende; su profundidad, por la diversidad de tamaños, clases y modelos que se ofrecen en cada línea de productos. En este contexto, de acuerdo con la entrevista realizada a Marienfeld, G. (comunicación personal, 22 de julio 2015) para la industria bajo estudio, podemos establecer la siguiente mezcla:

Tabla 7: Estructura de la mezcla de productos en el mercado de productos para laboratorio

\begin{tabular}{|c|c|c|c|c|}
\hline \multirow{2}{*}{\multicolumn{2}{|c|}{$\begin{array}{l}\text { DIMENSIONES DE LA } \\
\text { MEZCLA }\end{array}$}} & \multicolumn{3}{|c|}{$\begin{array}{c}\text { AMPLITUD } \\
\text { (Líneas de productos) }\end{array}$} \\
\hline & & Materiales & Equipos & Reactivos \\
\hline \multirow{4}{*}{ PROFUNDIDAD } & \multirow{4}{*}{$\begin{array}{l}\text { Diversos tamaños, } \\
\text { colores, modelos, } \\
\text { calidad, pesos, } \\
\text { forma, } \\
\text { composición y } \\
\text { usos. }\end{array}$} & Vidrio & Livianos & Para Análisis (P.A.) \\
\hline & & Porcelana & Semipesados & $\begin{array}{l}\text { Para Uso } \\
\text { farmacéutico } \\
\text { (U.S.P.) }\end{array}$ \\
\hline & & Metal & Pesados & $\begin{array}{l}\text { Químicamente } \\
\text { Puros (Q.P.) }\end{array}$ \\
\hline & & Plástico & & Reactivos Clínicos \\
\hline
\end{tabular}

Fuente: adaptado de Stanton (2007).

Elaboración: propia.

A continuación, se detalla una muestra de los principales productos importados de acuerdo con su clasificación arancelaria y el país de procedencia de las importaciones, incluyendo montos acumulados y participación de mercado para el período 2001-2020. 
Tabla 8: Estadísticas de una muestra de productos importados para laboratorio por partidas arancelarias, país proveedor, valores acumulados 2001-2020 y participación (En miles de US\$)

\begin{tabular}{|c|c|c|c|c|}
\hline $\begin{array}{c}\text { Partida } \\
\text { arancelaria } \\
(*)\end{array}$ & Descripción & País proveedor & $\begin{array}{c}\text { Valores } \\
\text { acumulados } \\
\mathbf{2 0 0 1 - 2 0 2 0}\end{array}$ & $\begin{array}{c}\text { Participación de } \\
\text { mercado } \\
(\%)^{* *}\end{array}$ \\
\hline \multirow[t]{5}{*}{382200} & \multirow{5}{*}{$\begin{array}{l}\text { Reactivos para } \\
\text { diagnóstico o laboratorio }\end{array}$} & Estados Unidos & 181,384 & 33.36 \\
\hline & & Alemania & 109,334 & 20.11 \\
\hline & & China & 60,536 & 11.13 \\
\hline & & Reino Unido & 24,579 & 4.52 \\
\hline & & Otros & 167,844 & 30.87 \\
\hline \multirow[t]{4}{*}{690911} & \multirow{4}{*}{$\begin{array}{l}\text { Aparatos y artículos para } \\
\text { uso químico o técnico de } \\
\text { porcelana }\end{array}$} & Estados Unidos & 732 & 30.78 \\
\hline & & China & 437 & 18.38 \\
\hline & & Alemania & 410 & 17.24 \\
\hline & & Otros & 799 & 33.60 \\
\hline \multirow[t]{4}{*}{701790} & \multirow{4}{*}{$\begin{array}{l}\text { Material de vidrio para } \\
\text { laboratorio }\end{array}$} & China & 9,230 & 24.87 \\
\hline & & Estados Unidos & 9,081 & 24.47 \\
\hline & & Alemania & 6,589 & 17.76 \\
\hline & & Otros & 12,207 & 32.90 \\
\hline \multirow[t]{4}{*}{842119} & \multirow{4}{*}{$\begin{array}{l}\text { Centrífugas para } \\
\text { laboratorio }\end{array}$} & Alemania & 66,825 & 42.71 \\
\hline & & Estados Unidos & 13,756 & 8.79 \\
\hline & & China & 10,755 & 6.87 \\
\hline & & Otros & 65,132 & 41.63 \\
\hline \multirow[t]{5}{*}{901180} & \multirow[t]{5}{*}{ Microscopios ópticos } & Alemania & 20,419 & 30.96 \\
\hline & & China & 15,411 & 23.36 \\
\hline & & Estados Unidos & 10,604 & 16.08 \\
\hline & & Japón & 8,246 & 12.50 \\
\hline & & Otros & 11,279 & 17.10 \\
\hline \multirow[t]{4}{*}{901600} & \multirow{4}{*}{$\begin{array}{l}\text { Balanzas electrónicas de } \\
\text { precisión }\end{array}$} & Suiza & 8,383 & 29.00 \\
\hline & & China & 7,144 & 24.72 \\
\hline & & Alemania & 6,657 & 23.03 \\
\hline & & Otros & 6,718 & 23.24 \\
\hline
\end{tabular}

(*) Código del Sistema Armonizado de Designación y Codificación de Mercancías (OMA, 1983).

**Participación de mercado acumulada 2001-2020.

Fuente: elaborada con datos de http://www.trademap.org

La tabla refleja el consolidado de las exportaciones hacia Perú, de una muestra de los principales productos para laboratorio según partida arancelaria, país, valores y participación de mercado acumulada por país proveedor. Podemos comentar que en el caso de la partida 382200, el abastecimiento ha sido siempre dominado por los Estados Unidos y Alemania, quienes en conjunto siempre han superado el 50\% de participación del mercado. En el caso de la partida 690911 (660911), la situación ha cambiado y China se ha convertido en el segundo proveedor desplazando a Alemania al tercer lugar. La partida 701790 ha tenido cambios en el nivel de abastecimiento, desde el año 2017 China ha tomado el liderazgo en el aprovisionamiento, relegando a Estados Unidos. La partida 842119 es suministrada principalmente por Alemania quien domina el mercado de proveedores por variables como calidad, marca y posicionamiento. En el caso de la partida 901180, el mercado de proveedores está cambiando y China ha tomado la segunda posición, modificando la rivalidad competitiva entre Alemania, Estados Unidos y Japón. Respecto a la partida 901600, es Suiza domina el suministro con una participación del 29\%, basada en el fuerte 
posicionamiento de sus marcas, le siguen China y Alemania con $24.72 \%$ y $23.03 \%$ de participación, respectivamente.

Existen líneas de productos cuyo suministro se encuentra altamente concentrado en pocos países, especialmente para las partidas 901180 (cuatro países poseen el 82.9\%) y 901600 (tres países acumulan el 76.75\%). Sin embargo, el mercado seguirá cambiando. La tendencia se orienta hacia una relativa desconcentración de países suministradores con destino al Perú, lo cual representa una oportunidad para las empresas importadoras. China está aumentando gradualmente su participación de mercado en el aprovisionamiento de productos para laboratorio hacia Perú por la capitalización de oportunidades. Sin embargo, es interesante conocer la opinión del empresario alemán Marienfeld, G. (comunicación personal, 21 mayo 2021), quien afirma que en Alemania las compañías pagan impuestos altos, tienen elevados costos de personal y no están subvencionados por su gobierno ni por bajos salarios. Cuentan una estricta legislación laboral, seguridad en el trabajo y regulaciones severas relacionadas con la conservación del medioambiente, lo cual cuesta mucho más que en China.

\subsection{CANALES DE DISTRIBUCIÓN EN LA INDUSTRIA}

Según Lambin (2003), canal de distribución es una estructura compuesta por socios independientes quienes participan en el proceso de colocar los bienes y servicios a disposición de los consumidores o usuarios industriales. En la industria bajo estudio, se trata de un canal de distribución internacional que puede explicarse mejor con el aporte de Minervini (2004) quien nos da definiciones para delinear gráficamente el canal, él define al agente de ventas como "un ejecutivo que desarrolla contactos de venta, teniendo como sede principal el país del propio exportador”. Asimismo, el mismo autor, concibe al distribuidor como una persona jurídica (pocas veces es una persona física) quien compra el producto a su propio nombre, custodia la mercancía y la vende a terceros aplicado un margen, con el que cubre los gastos y obtiene la ganancia necesaria. Seguidamente, se muestra el canal de distribución del mercado bajo estudio.

Figura 1: Canal de distribución en el mercado peruano de venta de productos para laboratorio

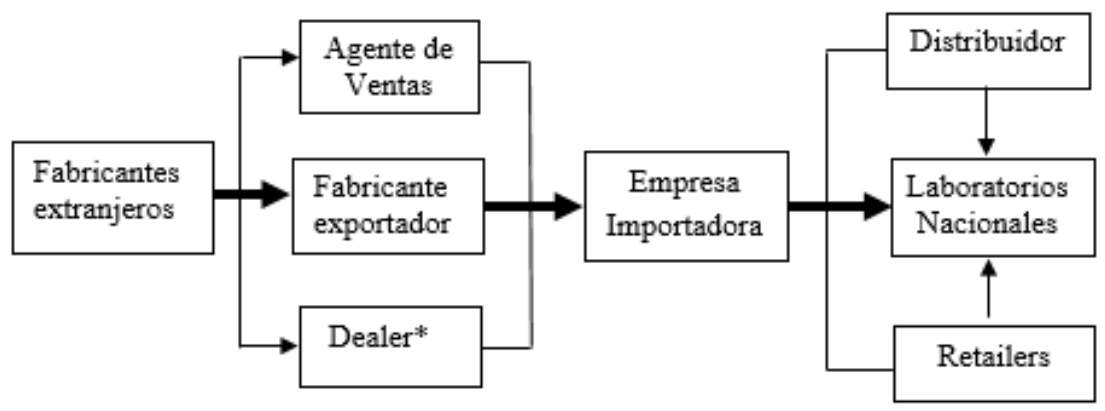

*Dealer: es un intermediario en el mercado exportador.

Elaboración: propia. 
El canal de distribución muestra que los fabricantes, en la mayoría de los casos, desempeñan la función de exportadores. Las exportaciones de China se dan usualmente mediante distribuidores o trading companies. Por otro lado, los importadores locales realizan ventas directas a las empresas que compran productos para laboratorio, esto se da en el caso que los clientes son empresas importantes. También venden mediante distribuidores, algo usual en ventas a provincias tales como Arequipa y Trujillo. Además, existe un mercado semi-formal en el centro de Lima donde hay comerciantes que venden al por menor (retailers), quienes logran abastecer marginalmente a algunos laboratorios.

\section{RESULTADOS Y DISCUSIÓN}

Basados en el enfoque del análisis de contenido y la información proveniente de fuentes primarias y secundarias, tenemos:

El mercado importador de productos para laboratorio es un mercado industrial porque las firmas importadoras no venden directamente a consumidores o usuarios, o sea, no abastecen a mercados de consumo masivo, sino más bien suministran materiales, equipos y reactivos químicos a empresas que los usan para producir bienes, prestar servicios o que los revenden a otras empresas.

De acuerdo con la información obtenida de la Oficina Nacional de Registros Públicos y la Superintendencia Nacional de Aduanas y Administración Tributaria (SUNAT), podemos decir que el mercado importador de productos para laboratorio comenzó a operar formalmente hace 71 años aproximadamente, con la fundación de la empresa H.W. Kessel en 1950. Posteriormente, otras empresas han ingresado al mercado, siendo 59 años el promedio de operaciones de las cinco principales firmas de la industria.

Las variables de mayor relevancia del entorno general que influyen en el desempeño de las empresas importadoras de productos para laboratorio son las variables políticas, económicas y las legales, a las cuales podemos incorporar también las variables tecnológicas, dado el nuevo escenario en que operan los negocios y las necesidades impostergables de digitalización empresarial.

Considerando el entorno sectorial puede decirse que las barreras de ingreso al mercado bajo estudio, son relativamente altas y están representadas por la inversión requerida, el conocimiento de los productos, la experiencia en este tipo de negocio y la lealtad a la marca que desarrollan los jefes y usuarios de laboratorios.

Los principales países proveedores de productos para laboratorio, a nivel global, en la producción y exportación de materiales (vidrio, metal. Porcelana y plástico) son Alemania y los Estados Unidos mientras que en la fabricación de equipos son Alemania, Japón y los Estados Unidos. Por otro lado. El 
líder en la elaboración y exportación de reactivos químicos es Estados Unidos. El liderazgo de estos países se sustenta en el aprendizaje logrado mediante la experiencia, la calidad y la innovación.

Las ventas e importaciones de las cinco principales empresas del mercado importador de productos para laboratorio durante el período 2000-2020, muestran una tendencia creciente, basada en el crecimiento económico sostenible que ha mostrado Perú en el mismo período, con excepción del año 2020 en que la pandemia del coronavirus generó una recesión económica mundial. Un factor favorable también fue la variable legal que fomentó una facilitación del comercio mundial y una economía de libre mercado.

Las estadísticas 2001-2020 de una muestra de productos para laboratorio, revela que las centrífugas para laboratorio (42.7\%) y los microscopios ópticos, son suministradas en mayor proporción por Alemania (41.6\%), mientras que las balanzas electrónicas de precisión las abastece principalmente Suiza (29.0\%). La tradición fabril y el know how, combinados con la calidad y capacidad innovadora son la fuente de ventaja competitiva.

El principal proveedor de reactivos para diagnóstico o laboratorio durante el período 2001-2020 es Estados Unidos (33.4\%) mientras que el material de vidrio para laboratorio es abastecido en mayor proporción por China (24.9\%) y Estados Unidos (24.5\%). Esta situación se explica porque los fabricantes chinos han mejorado sus procesos productivos, los costos de fabricación y la calidad del producto final.

Respecto a las prácticas comerciales sobre la modalidad de cobranza a los importadores de productos para laboratorio que forman parte de su cartera de clientes, Marienfeld, G. (comunicación personal, 21 mayo 2021), refiere que sus clientes llevan décadas trabajando con ellos y que les gusta pagar mediante prepago porque se ahorran los gastos de la carta de crédito y además obtienen un descuento por pronto pago. En los países con una seguridad jurídica elevada también venden contra cuenta abierta, esto significa con facturas a plazo. Evidentemente, la base de todo negocio es la confianza entre las partes.

Sobre los efectos relevantes que la pandemia ha ocasionado en el desempeño del negocio, Marienfeld, G. (comunicación personal, 21 mayo 2021), manifiesta que algunos de sus clientes domiciliados en países muy afectados por la pandemia, han reducido los volúmenes y valor de los pedidos. Por otra parte, en el caso de su empresa, ellos no tuvieron que despedir a nadie y mantuvieron a sus trabajadores ocupados, cumpliendo con las regulaciones del gobierno que les exige proporcionar a sus empleados pruebas de COVID-19 así como máscaras para cubrir bocas y narices, guardando la distancia al realizar trabajo presencial en la fábrica.

Acerca del futuro de la industria de productos para laboratorio a nivel global, Marienfeld, G. (comunicación personal, 21 mayo 2021), espera que las vacunas e infecciones creen una inmunidad de rebaño en todo el mundo, para que la carga de trabajo vuelva a la normalidad. No tiene certeza de cómo va a cambiar el negocio a nivel global y tiene pocas esperanzas que China haga una competencia más 
justa en el futuro. Afirma que, hasta hoy, su ventaja competitiva es la mayor calidad, fiabilidad y la rápida tramitación de negocios.

Sobre los efectos relevantes que la pandemia ha ocasionado en el desempeño de los negocios, Navas, C. (comunicación personal, 16 diciembre 2021), señala que los niveles de venta se han contraído parcialmente porque los clientes han asumido conductas de compra más conservadoras, los procedimientos de venta y atención al cliente se complican por la falta de interacción humana, las empresas asumen mayores gastos por la implementación de programas de digitalización y que los costos de compra han aumentado. Esto se corrobora con los resultados de las ventas anuales y los valores importados en el año 2020 y los desafíos de la automatización de operaciones.

En el contexto de los recursos humanos, Navas, C. (comunicación personal, 16 diciembre 2021), opina que las empresas han reducido parcialmente sus planillas de empleados para enfrentar situaciones críticas por la pandemia, que la cultura organizacional fue afectada negativamente por los temores de la pandemia y que las normas sanitarias para enfrentar la COVID-19 pueden influir negativamente en la rentabilidad de las firmas importadoras. Una de las primeras decisiones tomadas por varios empresarios peruanos, fue reducir los gastos de personal y subcontratar actividades que no contribuyen con la creación de valor.

En general, podemos afirmar que la pandemia del coronavirus ha modificado la estrategia general de las firmas importadoras de productos para laboratorio en el Perú. En este contexto, las empresas, buscan implementar estrategias mixtas de diferenciación, reducción de costos y enfoque. Finalmente, consideramos que es necesario realizar nuevas investigaciones, incluyendo una muestra más amplia de empresas y directivos entrevistados, que represente a empresas con experiencia, reputación y sean contribuyentes importantes, para lograr un mayor alcance y profundidad en los resultados. 


\section{REFERENCIAS}

-Banco Central de Reserva del Perú. (2021). Recuperado de https://www.bcrp.gob.pe

-David, F. (2013). Conceptos de administración estratégica. Decimocuarta edición. Editorial Pearson Educación.

-Ipsos Perú. (2021). Base de datos del Directorio de Instituciones, Medios y Empresa-DIME (2021). Recuperado de https://www.dime.pe

-Guimbert, X. (2010). Pensar estratégicamente: modelos conceptos y reflexiones. Editorial Deusto S.A.

-Hernández, R.; Fernández, C. y Baptista, M. (2010). Metodología de la investigación. Quinta edición. Editorial McGraw Hill-Interamericana Editores.

-Hill, Ch. y Jones, G. (2011). Administración estratégica: un enfoque integral. Editorial Cengage Learning.

-Hitt. M.; Ireland, D. \& Hoskisson, R. (2017). "Strategic management: competitiveness and globalization, concepts and cases". $12^{\text {th }}$ edition. Cengage Learning.

-International Chamber of Commerce (2019), Incoterms 2020: ICC rules for the use of domestic and international trade terms. ICC Publishing.

-Johnson, G.; Scholes, K. y Whittington, R. (2006). Dirección estratégica. Séptima edición

-Kotler, P. y Keller, K. (2012). Dirección de marketing. Decimocuarta edición. Editorial Pearson Educación.

-Krippendorff, K. (1990). Metodología del análisis de contenido. Ediciones Paidós Ibérica.

-Lambin, J. (2003). Marketing estratégico. Editorial ESIC.

-Magretta (2012). Understanding Michael Porter: the essential guide to competition and strategy. Harvard Business Review Press. Boston.

-Minervini, N. (2004). La ingeniería de la exportación. Cuarta edición. Editorial McGraw-Hill.

-Normas legales. Diario Oficial El Peruano. Decreto Legislativo 668 publicado el 11 de setiembre de 1991. Perú.

-Normas legales. Diario Oficial El Peruano. Decreto Legislativo 1053 (Ley General de Aduanas), publicado el 27 de junio de 2008. Perú.

-Normas legales. Diario Oficial El Peruano. Ley 30056 (Ley de impulso al desarrollo productivo y crecimiento empresarial), publicada el 02 de julio de 2013. Perú.

-Normas legales. Diario Oficial El Peruano. Ley 30296 (Ley que promueve la reactivación de la economía), publicada el 31 de diciembre de 2014. Perú.

-Normas legales. Diario Oficial El Peruano. Decreto Supremo 044-2020-PCM. Publicado el 15 de marzo de 2014. Perú.

-Normas legales. Diario Oficial El Peruano. Decreto Supremo No 380-2019-EF, publicado el 20 de diciembre de 2019. Perú. 
-Organización Mundial de Aduanas-OMA (1983). Sistema Armonizado de Designación y Codificación de Mercancías. Bélgica.

-Peru Top Publications. (2021). Top Online Database. Recuperado de http://www.ptp.pe

-International Trae Centre. (2021). Database. https://www.trademap.org

-Porter, M. (1982). Estrategia competitiva: técnicas para el análisis de los sectores industriales y de la competencia. Editorial CECSA.

-Salkind, N. J. (1998). Método de investigación. Prentice-Hall.

-Stanton, W.; Etzel, M. y Walker, B. (2007). Fundamentos de marketing. Editorial McGraw-Hill Interamericana.

-Superintendencia de Banca y Seguros. (2021). Recuperado de https://www.sunat.gob.pe

-Superintendencia Nacional de Aduanas y de Administración Tributaria. (2021). Recuperado de https://www.sunat.gob.pe

-Superintendencia Nacional de Registros Públicos SUNARP (2015). Lima, Perú.

-Thompson, A.; Gamble, J y Peteraf, M. (2015). Essentials for strategic management: the quest for competitive advantage. Fourth edition. McGraw-Hill Education.

-Trading Economics. (2022). Database. Recuperado de https://www.tradingeconomics.com 


\section{ANEXO}

Anexo 1: Criterios de selección de los entrevistados

\begin{tabular}{|l|l|l|l|}
\hline \multicolumn{1}{|c|}{$\begin{array}{c}\text { Entrevistados } \\
\text { Criterios }\end{array}$} & \multicolumn{1}{|c|}{ Gerd Marienfeld } & \multicolumn{1}{c|}{ Carla Navas } & \multicolumn{1}{c|}{ Jesús Valencia } \\
\hline Empresa & $\begin{array}{l}\text { Paul Marienfeld } \\
\text { GmbH + Co. KG }\end{array}$ & H.W. Kessel S.A.C. & Diproquim S.R.L. \\
\hline Condición & Exportador & Importador & Distribuidor \\
\hline Experiencia & Desde 1922 & Desde 1950 & Desde 1992 \\
\hline Cargo desempeñado & Gerente general & Gerente de producto & Gerente general \\
\hline Tiempo de servicios & 33 & 12 & 29 \\
\hline Grado académico & Ing. Industrial & Lic. en Química & Ing. Químico \\
\hline Accesibilidad & Alta & Alta & Alta \\
\hline Ubicación & Alemania & Lima & Arequipa \\
\hline Confiabilidad & $\begin{array}{l}\text { ISO 9001 otorgada por } \\
\text { DIN EN }\end{array}$ & $\begin{array}{l}\text { Número obtenido de } \\
\text { Representaciones }\end{array}$ & $\begin{array}{l}\text { Personalización } \\
\text { servicio }\end{array}$ \\
\hline
\end{tabular}

Elaboración: propia.

Anexo 2: Indicadores macroeconómicos clave, Perú, 2001-2020

\begin{tabular}{|c|c|c|c|c|}
\hline Años & $\begin{array}{c}\text { PBI } \\
\text { (Variación \%) }\end{array}$ & $\begin{array}{l}\text { Tipo de Cambio } \\
\text { Promedio anual }\end{array}$ & $\begin{array}{c}\text { Inflación anual } \\
\text { Promedio }(\%)\end{array}$ & $\begin{array}{c}\text { Tasa de Interés } \\
\text { Activa M/N (\%)* }\end{array}$ \\
\hline 2001 & 0.6 & S/. 3,51 & 2.0 & 23.39 \\
\hline 2002 & 5.5 & S/. 3,52 & 0.2 & 20.19 \\
\hline 2003 & 4.2 & S/. 3,48 & 2.3 & 23.30 \\
\hline 2004 & 5.0 & S/. 3,41 & 3.7 & 25.98 \\
\hline 2005 & 6.3 & S/. 3,30 & 1.6 & 23.48 \\
\hline 2006 & 7.5 & S/. 3,27 & 2.0 & 23.80 \\
\hline 2007 & 8.5 & S/. 3,13 & 1.8 & 22.39 \\
\hline 2008 & 9.1 & S/. 2,93 & 5.8 & 23.12 \\
\hline 2009 & 1.1 & S/. 3,01 & 2.9 & 20.04 \\
\hline 2010 & 8.3 & S/. 2,83 & 1.5 & 18.78 \\
\hline 2011 & 6.3 & S/. 3,34 & 3.4 & 19.11 \\
\hline 2012 & 6.1 & S/. 2,64 & 3.7 & 19.06 \\
\hline 2013 & 5.9 & S/. 2,70 & 2.8 & 16.02 \\
\hline 2014 & 2.4 & S/. 2,84 & 3.2 & 16.26 \\
\hline 2015 & 3.3 & S/. 3,19 & 3.5 & 16.23 \\
\hline 2016 & 4.0 & S/. 3,38 & 3.6 & 17.91 \\
\hline 2017 & 2.5 & S/. 3,26 & 2.8 & 15.78 \\
\hline 2018 & 4.0 & S/. 3,29 & 1.3 & 14.37 \\
\hline 2019 & 2.2 & S/. 3,34 & 2.1 & 14.35 \\
\hline 2020 & -11.0 & S/. 3,50 & 1.8 & 12.10 \\
\hline
\end{tabular}

*Tasa de interés activa anual en moneda nacional del sistema bancario.

Fuente: elaborada con datos de http://www.bcrp.gob.pe y http://www.sbs.gob.pe 